\title{
The Effect of Diet on Intermediary Metabolite Concentrations in Type 2 (Non-Insulin-Dependent) Diabetes Mellitus
}

\author{
M.C. Sheppard, J. Burrin, K.G. M. M. Alberti and M. Nattrass \\ General Hospital, Birmingham and Department of Clinical Biochemistry, Royal Victoria Infirmary, Newcastle-upon-Tyne, UK
}

\begin{abstract}
Summary. The metabolic response to a standard meal was studied in six Type 2 (non-insulin-dependent) diabetic patients at diagnosis and following 4-6 weeks of dietary treatment. The fall in blood glucose concentration following treatment was accompanied by significant reductions in circulating concentrations of lactate, pyruvate, alanine and glycerol.
\end{abstract}

Blood 3-hydroxybutyrate concentrations also fell with treatment.

Key words: Type 2 (non-insulin-dependent) diabetes, diet, blood glucose, lactate, pyruvate, alanine, glycerol, 3-hydroxybutyrate.
Despite considerable controversy concerning the most appropriate diet for treating patients with Type 2 (noninsulin-dependent) diabetes mellitus [1, 2], the role of carbohydrate and/or calorie restriction in correcting hyperglycaemia is well established [3]. Little attention has been paid, however, to abnormalities of other intermediary metabolites in untreated Type 2 diabetic patients and the response of these to treatment. In previous studies of Type 2 diabetic patients treated with sulphonylureas [4] or biguanide therapy [5, 6], abnormalities of circulating intermediary metabolite concentrations were found, although the therapeutic and prognostic importance of these abnormalities is not known [7]. We have studied the response of blood concentrations of the gluconeogenic precursors, lactate, pyruvate, alanine and glycerol, and the ketone body, 3-hydroxybutyrate, in Type 2 diabetic patients for $4 \mathrm{~h}$ following a standard breakfast, at diagnosis and following correction of hyperglycaemia by a period of diet.

\section{Patients and Methods}

\section{Patients}

Six newly diagnosed male Type 2 diabetic patients were studied. All had polydipsia and polyuria, with a random blood glucose $>12$ $\mathrm{mmol} / \mathrm{l}$ at diagnosis. None had evidence of hepatic or renal disease on routine biochemical screening and none were taking drugs known to affect carbohydrate metabolism. Subjects were considered suitable for initial treatment by diet on the basis of little or no weight loss and absence of ketonuria at presentation. The protocol of the study was approved by the Ethical Committee of the Central Birmingham Health District and informed consent was obtained. Mean age of patients was 55 years (range 42-65 years) and mean weight $87 \mathrm{~kg}$ (range $78-102 \mathrm{~kg}$ ) at diagnosis; all were over $120 \%$ desirable body weight [8].

\section{Protocol}

After an overnight fast an indwelling Teflon cannula was inserted into an antecubital vein at $0800 \mathrm{~h}$. Two fasting samples were taken at $0825 \mathrm{~h}$ and $0830 \mathrm{~h}$, after which a standard $40 \mathrm{~g}$ carbohydrate breakfast was eaten. Blood samples were taken half-hourly for $4 \mathrm{~h}$. Blood for metabolite assays $(1-2 \mathrm{ml})$ was mixed with $5 \mathrm{ml}$ of $5 \%(\mathrm{v} / \mathrm{v})$ ice-cold perchloric acid and refrigerated immediately. Glucose (hexokinase method), lactate, pyruvate, glycerol, alanine and 3-hydroxybutyrate were assayed in the perchloric acid extract by automated enzymic fluorimetric methods [9].

\section{Diet}

The standard meal stimulus consisted of $80 \mathrm{~g}$ bread ( $40 \mathrm{~g}$ of carbohydrate) and butter with a boiled egg (weight $60 \mathrm{~g}$ ). One cup of tea was drunk with the meal containing $20 \mathrm{ml}$ of milk. The meal was started immediately after withdrawal of the second fasting sample and eaten within $10 \mathrm{~min}$. Following the blood sample at $1000 \mathrm{~h}$ subjects drank a cup of coffee containing $20 \mathrm{ml}$ of milk and ate two biscuits $(30 \mathrm{~g}$ weight, containing $20 \mathrm{~g}$ carbohydrate).

Patients were prescribed a diet following an initial dietary assessment of previous calorie intake by a dietitian. Those in whom previous dietary intake was in the range $1000-2000 \mathrm{kcal}$ were prescribed $1000 \mathrm{kcal}$ diets. When previous dietary intake exceeded $2000 \mathrm{kcal}$ patients were prescribed diets containing $1500 \mathrm{kcal}$. The moderate calorie restriction was accompanied by advice to exclude refined sugars from the diet. The approximate carbohydrate concentration of the diet was $40 \%$. 
Table 1. Fasting and 4-h mean concentrations of blood intermediary metabolites and serum insulin before and after dietary treatment in six Type 2 diabetic patients

\begin{tabular}{|c|c|c|c|c|}
\hline & \multicolumn{2}{|c|}{ Fasting concentration } & \multicolumn{2}{|c|}{ 4-h mean concentration } \\
\hline & Diagnosis & After diet & Diagnosis & After diet \\
\hline \multicolumn{5}{|l|}{ Metabolite (mmol/l) } \\
\hline Lactate & $1.08 \pm 0.12$ & $0.71 \pm 0.07^{a}$ & $1.05 \pm 0.02$ & $0.74 \pm 0.03^{b}$ \\
\hline Pyruvate & $0.09 \pm 0.01$ & $0.05 \pm 0.01^{\mathrm{a}}$ & $0.09 \pm 0.01$ & $0.06 \pm 0.01^{b}$ \\
\hline Alanine & $0.28 \pm 0.03$ & $0.25 \pm 0.03^{\mathrm{a}}$ & $0.29 \pm 0.01$ & $0.25 \pm 0.01^{b}$ \\
\hline Insulin (mU/1) & $12.6(1.0-27.1)$ & $8.4(4.1-14.6)$ & $23.6(5.8-45.8)$ & $24.8(16.0-46.5)$ \\
\hline
\end{tabular}

Results expressed as mean \pm SEM for metabolites and mean (range) for insulin. Significance was sought using Student's paired t-test for fasting samples and two-way analysis of variance for 4 -h responses. ${ }^{\mathrm{a}} p<0.05 ;{ }^{\mathrm{b}} p<0.01$

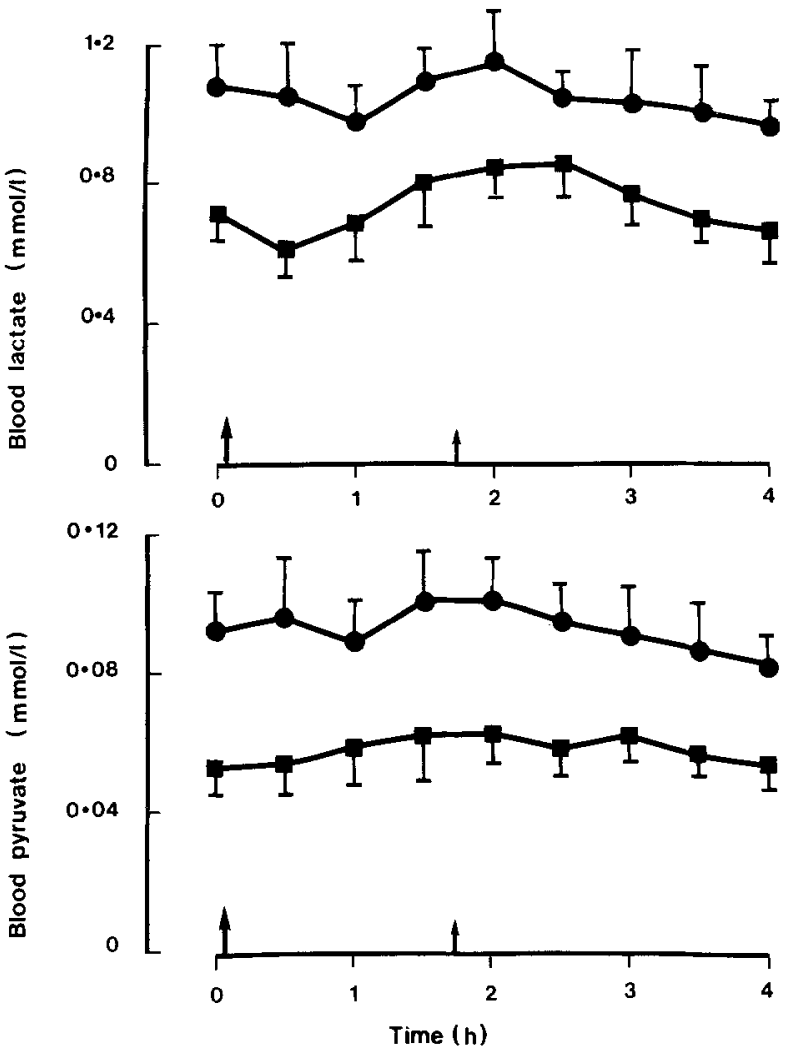

Fig. 1. Blood lactate and blood pyruvate concentrations (mean \pm SEM) following a standard breakfast in six Type 2 diabetic patients before (- $)$ ) and after ( $\square$ - a period of dietary treatment. The large arrow indicates breakfast and the small arrow a snack

\section{Statistical Analysis}

Results are presented as mean \pm SEM. The nine values for each metabolite or hormone over the 4-h period in any individual patient have been averaged and this is referred to as the $4 \mathrm{~h}$ mean. Significance has been calculated using Student's paired t-test for fasting samples and two-way analysis of variance for values for each patient over the sampling period before and after treatment.

\section{Results}

All patients showed a satisfactory clinical response as judged by loss of symptoms, loss of glycosuria, and a

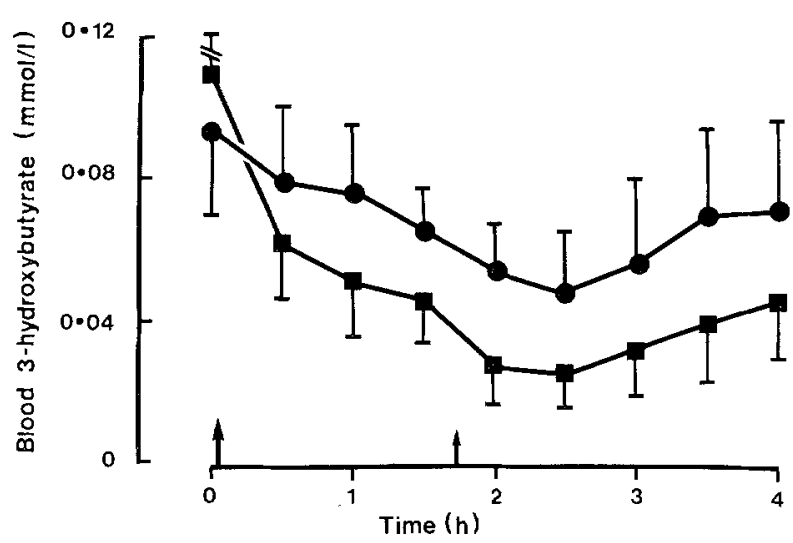

Fig. 2. Blood 3-hydroxybutyrate concentrations (mean \pm SEM) following a standard breakfast in six Type 2 diabetic patients before (-) and after ( - a period of dietary treatment. The large arrow indicates breakfast and the small arrow a snack

fall in post-prandial blood glucose of a least $5 \mathrm{mmol} / 1$ (range: $5.0-22.4 \mathrm{mmol} / 1$ ). Mean weight loss was $3.5 \mathrm{~kg}$ (range: $0.5-7.0 \mathrm{~kg}$ ) and no patient gained weight. Mean fasting blood lactate concentration was elevated at diagnosis but fell with treatment $(p<0.05$, Table 1, Fig. 1$)$. A similar fall was seen following treatment for 4-h mean lactate concentration $(p<0.01)$ and for mean fasting pyruvate $(p<0.05)$, alanine and glycerol, and 4 -h mean pyruvate, alanine and glycerol (all $p<0.01$ ). Ketone body concentrations were within the normal range. No difference was observed in fasting values, although 4-h mean values were significantly different $(p<0.01$, Table 1). Concentrations fell post-prandially, rising again towards the end of the sampling period (Fig. 2). The difference between the two studies lies in the rate of fall of 3 -hydroxybutyrate in the first $30 \mathrm{~min}$.

\section{Discussion}

All patients lost symptoms and glycosuria and showed a fall in post-prandial blood glucose concentration. This occurred concomitant with modest weight reduction. The most striking finding was the small but consistent 
and significant fall in 4-h mean concentrations of intermediary metabolites. The mechanism by which intermediary metabolite concentrations are reduced by diet is unclear. Increased glycolysis in muscle, or enhanced inhibition of gluconeogenesis would be expected to increase blood concentrations of lactate, pyruvate and alanine. The fall in these metabolites, concomitant with the fall in glucose implies intracellular diversion to other fates, either pyruvate oxidation in tissues such as muscle, or into glycogen in liver. The fall in 3-hydroxybutyrate concentration is of interest since dietary restriction might be expected to increase lipolysis and hence ketogenesis. The results suggest that lipolytic inhibition is enhanced which is further supported by the fall in glycerol.

\section{References}

1. Mann JI (1980) Diet and diabetes. Diabetologia 18: 89-95

2. Reaven GM (1980) How high the carbohydrate? Diabetologia 19: 409-413

3. Bloom A (1977) Some practical aspects of the management of diabetes. Clin Endocrinol Metab 6: 399-417
4. Nattrass M (1980) Metabolic rhythms during sulphonylurea therapy. Diabetologia 19: 301-302

5. Nattrass M, Todd PG, Turnell D, Alberti KGMM (1978) Metabolic abnormalities during combined sulphonylurea and phenformin therapy in maturity-onset diabetics. Diabetologia 14: 389-395

6. Nattrass M, Hinks L, Smythe P, Todd PG, Alberti KGMM (1979) Metabolic effects of combined sulphonylurea and metformin therapy in maturity-onset diabetics. Horm Metab Res 11:332-337

7. Alberti KGMM, Hockaday TDR (1975) The biochemistry of the complications of diabetes mellitus. In: Keen H, Jarrett J (eds) Complications of Diabetes. Edward Arnold, London, pp 221-264

8. Desirable weights of adults (1959) Metropolitan Life Insurance Company Statistical Bulletin 40:824

9. Lloyd B, Burrin J, Smythe P, Alberti KGMM (1978) Enzymic fluorimetric continuous-flow assays for blood glucose, lactate, pyruvate, alanine, glycerol, and 3-hydroxybutyrate. Clin Chem 24: $1724-1729$

Received: 24 March 1982

and in revised form: 17 January 1983

Dr. M. Nattrass

The General Hospital

Steelhouse Lane

Birmingham B4 6NH, UK 